\title{
Evaluation of Bioactive Compounds in Moringa oleifera Flower Using Gas Chromatography Mass Spectrometry/Fourier Transform Infrared Spectroscopy: The Need for Good Postharvest Handling
}

\author{
Amaechi NC* \\ Department of Food Science and Technology, Abia State University, Uturu-Abia \\ State, Nigeria \\ *Corresponding Author: Amaechi NC, Department of Food Science and \\ Technology, Abia State University, Uturu-Abia State, Nigeria.
}

DOI: $10.31080 /$ ASNH.2020.05.0967
Received: November 01, 2021

Published: November 29, 2021

(C) All rights are reserved by Amaechi NC.

\begin{abstract}
Moringa oleifera flower is consumed as an edible flower by some human populations. The effect of postharvest handling was evaluated by identifying hydrophilic and hydrophobic bioactive compounds in flowers kept at ambient temperature in a cellophane paper bag and in freshly harvested flowers. Bioactive compounds were extracted using methanol and dichloromethane/methanol and the extracts were subjected to gas chromatography/mass spectrometry to separate and identify bioactive compounds. Fourier transform infrared spectroscopy was used to show functional groups of bioactive compounds in the various extracts. The most abundant compounds from the methanol extract of the flowers kept at ambient temperature were 1,3-Butadiene-1-carboxylic acid, DAllose and 1,12-Tridecadiene while the most abundant compounds from the dichloromethane/methanol extract were hydrazine-1,1dimethyl, acetic acid, urea and isobutylamine. The most abundant compounds from dichloromethane/methanol extract of freshly harvested flowers were hexadecanoic acid methyl ester, 9,12-Octadecadienoic acid methyl ester and 10,13-Octadecadienoic acid methyl ester. Fourier transform infrared spectroscopy confirmed the presence of amine, aldehyde, alkane, hydroxyl, methoxy, methyl ether, carboxylic acids, amide, organic nitrates and organic siloxane compounds. Compounds from the various extracts have been reported to have beneficial attributes for wellness which provides support for the use of Moringa oleifera flower as food. Postharvest handling in cellophane bag at ambient temperature resulted to the formation of compounds reported to be toxic such as being carcinogenic. Therefore, prior to processing such as drying, there is need for efficient postharvest handling.
\end{abstract}

Keywords: Moringa oleifera Flowers; Bioactive Compounds; Postharvest Handling; Gas Chromatography; Mass Spectrometry

\section{Introduction}

Edible flowers are commonly used in human nutrition and their consumption have increased in recent years [1]. For some human populations around the world, they form an integral part of human diet for various reasons which range from sensory impact, health benefits and culinary purposes. Phytochemicals are natural bioactive compounds found in plants. The benefits of plants are based on the fact that they contain bioactive compounds which have positive impact on health and longevity. Edible flowers are consumed fresh or processed using different drying methods. The safety of flowers consumed as food depends much on postharvest handling and storage. Changes may occur in the bioactive compounds in the harvested plant part between the point of harvest and prior to further processing such as drying. Flowers which are incorporated in human diets must be toxic free and have good nutritional composition [2]. Moringa oleifera (Lam) plant belongs to the family Moringaceae [3]. It is considered one of the world's most useful trees as almost every plant part which include the leaves, seeds, roots and flowers are used as food for human and animal consumption, medication and industrial purposes [4,5]. The flowers are fragrant yel- 
Evaluation of Bioactive Compounds in Moringa oleifera Flower Using Gas Chromatography Mass Spectrometry/Fourier Transform Infrared Spectroscopy: The Need for Good Postharvest Handling

lowish white, bisexual borne in 10 to $25 \mathrm{~cm}$ long axillary, compound inflorescence known as the panicles. [6-8]. Amino acids evaluation indicated that the flowers have higher amino acids content than the leaves and seeds [9]. Water accounts for more than $80 \%$ as the main ingredient in flowers [10]. The high moisture content of fresh flowers can lead to quick deterioration either by microbial activities or biochemical changes induced by enzymes following postharvest handling at ambient temperature.

Analytical tools such as the use of gas-chromatography/mass spectrometry, high pressure liquid chromatography-diode array dictator electroscopy, high pressure liquid chromatography-diode array dictator- ESI/MS/MS of bioactive compounds in various solvent extracts of Moringa oleifera flower has been reported [11]. GC-MS has found usefulness as a tool which qualifies for the effective separation of phytochemicals [12]. Fourier Transform Infrared (FTIR) determines the vibrations of bonds within chemical functional groups and generates a spectrum that can be considered as a biochemical or metabolic "fingerprint" of the sample [13]. FTIR and GC-MS techniques have become firmly established as key technological metabolic profiling of medicinal plants [14]. The main research interest in plants is aimed at unveiling the presence of some active components in them [15]. Methanol solvent has best extractive ability for different primary and secondary metabolites from plants and pave a way for functional groups analysis by FTIR and separation studies by GC-MS analyses [16]. It is in view of this that GC/MS analysis was adopted to separate, identify and evaluate the effect of postharvest handling by identifying hydrophilic and hydrophobic bioactive compounds in freshly harvested flowers kept at ambient temperature in a cellophane paper bag and in harvested flowers that were processed immediately in the fresh state. Fourier transform infrared spectroscopy (FTIR) was used to identify functional groups to which the bioactive compounds belong to.

\section{Materials and Methods}

Sourcing of flower samples and extraction of bioactive compounds

Fresh Moringa oleifera flowers were harvested from a farmyard around Abia State University in Umudike Location (5²8'19.79' N; $7^{\circ} 32^{\prime} 33.59^{\prime \prime} \mathrm{E}$ ). Two portions of the freshly harvested samples were put in black cellophane bags and kept at room temperature $25 \pm$ $2^{\circ} \mathrm{C}$ ) for $6 \mathrm{~h}$. The third portion of the flower sample was processed in its fresh state immediately after harvest. Bioactive compounds in the various samples were extracted according to the method described by [17]. Each sample was pulverized and $10 \mathrm{~g}$ of each sample was put into labeled conical flasks respectively. Two hundred (200) $\mathrm{ml}$ methanol and $200 \mathrm{ml}$ dichloromethane/methanol $(1: 1, v / v)$ were added to conical flasks containing the respective samples, shaked vigorously and covered using aluminum foil. They were allowed to stand for $24 \mathrm{~h}$ at room temperature after which each sample mixture was filtered through No1 Whatman filter paper respectively. The extracts were concentrated by heating over a boiling water bath to remove excess solvent. The extracts were subjected to Gas Chromatography - Mass Spectrometry (GCMS) and Fourier Transform Infrared Spectroscopy (FTIR) analysis for the separation, identification and characterization of bioactive compounds.

Gas chromatography mass spectrometry analysis (GCMS)

GCMS analysis of each flower extract was done using Gas Chromatography-Mass Spectrometry (Agilent GG 7890B-MSD 5977A, Agilent Technology, USA). The gas chromatograph was equipped with capillary column of $30 \mathrm{~mm}$ length, $0.25 \mathrm{~mm}$ diameter and $20 \mathrm{~m}$ film thickness. Helium (99.99\%) was used as carrier gas. The temperature programming was set with initial column oven temperature of $50^{\circ} \mathrm{C}$, hold time of $3 \mathrm{~min}$ by $200^{\circ} \mathrm{C}$ to a final temperature of $325^{\circ} \mathrm{C}$ with hold time of $2 \mathrm{~min}$. $3 \mu \mathrm{l}$ of each Moringa oleifera flower extract was injected respectively using a Hamilton syringe into the gas chromatograph for total ion chromatographic analysis with split injection technique $(5: 1)$. The injector temperature was $25^{\circ} \mathrm{C}$ while for the mass spectrometer, the ion source temperature was $23^{\circ} \mathrm{C}$ with an interface temperature of $30^{\circ} \mathrm{C}$ and recorded over a scan range of 46 to $800 \mathrm{~m} / \mathrm{z}$ with electron impact ionization energy of $70 \mathrm{ev}$. Total run time of the gas chromatography-mass spectrometry analysis for the methanol and dichloromethane methanol extracts of the stored Moringa oleifera flowers was 64 and 69 min respectively while the total run time for dichloromethane/methanol extract of the freshly harvested and processed flowers was $69 \mathrm{~min}$.

\section{Fourier transform infrared spectroscopy (FTIR)}

FTIR of each extract was analyzed by the method described by [17]. A small quantity of each flower extract was dropped between two plates of sodium chloride and placed in the FTIR spectroscope chamber (Cary 630, Agilent Technologies, USA) respectively. Analysis was done in a scan range from $650-4000 \mathrm{~cm}$ with a resolution of $8 \mathrm{~cm}$. 
Evaluation of Bioactive Compounds in Moringa oleifera Flower Using Gas Chromatography Mass Spectrometry/Fourier Transform Infrared Spectroscopy: The Need for Good Postharvest Handling

\section{Results and Discussion}

Gas chromatography-Mass Spectrometry (GCMS) analysis of Moringa oleifera flower revealed the presence of 23 compounds in the methanol extract of fresh flowers stored at ambient temperature, 43 compounds in dichloromethane/methanol extract of freshly plucked and processed flower samples without storing at ambient temperature and 13 compounds in dichloromethane/ methanol extract of fresh flower sample stored at ambient temperature. Table 1 shows results of compounds identified in the methanol extract of freshly plucked Moringa oleifera flowers kept at ambient temperature in a cellophane bag. The most abundant compounds found in this extract were 1,3-Butadiene-1-carboxylic acid, D-allose and 1,12-Tridecadiene. These had relative abundance of $13.43,19.93$ and $29.78 \%$ respectively and representing $63.14 \%$ of the hydrophilic bioactive compounds. [18] reported the pres- ence of 26 compounds in methanol extract of shade dried Moringa oleifera flower grown in Tamilnadu, India for which oleate, quinic acid and Cis-9-hexadecenal were the major constituents. 1,3-Butadiene is an unsaturated fatty acid has been reported to have antiamyloid, Beta 2-receptor agonist and ß-glucuronidase inhibitory activity [19]. D-allose is an aldohexose sugar and a C-3-epimer of glucose. D-allose has been reported to have antioxidative activity [20], it suppresses renal inflammation by attenuating cisplatininduced nephrotoxicity [21] and has anti-proliferative effects on prostate cancer cell lines [22]. 1,12-Tridecadiene is an alkadiene which is an unsaturated aliphatic hydrocarbon. It has been reported in Curcurbita maxima seed [23] and as one of the major components found in cuticular abdominal glands of Zonocerus variegatus [24]. No clear specific biological activity has been reported for 1,12-Tridecadiene, however similar analogues have been reported to have medicinal properties.

\begin{tabular}{|c|c|c|c|c|c|}
\hline $\begin{array}{l}\text { Peak } \\
\text { Number }\end{array}$ & $\begin{array}{l}\text { Retention } \\
\text { Time (Min) }\end{array}$ & Compound Name & $\begin{array}{c}\text { Relative } \\
\text { Abundance } \\
(\%)\end{array}$ & $\begin{array}{c}\text { Molecular } \\
\text { Formula }\end{array}$ & $\begin{array}{l}\text { Molecular } \\
\text { Weight }\end{array}$ \\
\hline 1 & 6.018 & 1,2-Dichlororethylhydroperoxide & 2.65 & $\mathrm{C}_{2} \mathrm{H}_{4} \mathrm{Cl}_{2} \mathrm{O}_{2}$ & 130.952 \\
\hline 2 & 6.367 & $\begin{array}{c}\text { 1H-1,3-benzimidazole-2-carboxylic acid, } \\
\text { 1-methyl }\end{array}$ & 0.98 & $\mathrm{C}_{9} \mathrm{H}_{8} \mathrm{~N}_{2} \mathrm{O}_{2}$ & 176.17 \\
\hline 3 & 6.678 & 4-Ethynyl-1-methyl pyrazole & 1.48 & $\mathrm{C}_{6} \mathrm{H}_{6} \mathrm{~N}_{2}$ & 106.128 \\
\hline 4 & 7.143 & Pent-2-ynal,4,4-dimethyl & 2.06 & $\mathrm{C}_{7} \mathrm{H}_{10} \mathrm{O}$ & 110.156 \\
\hline 5 & 7.996 & 1,3-Butadiene-1-carboxylic acid & 13.43 & $\mathrm{C}_{5} \mathrm{H}_{6} \mathrm{O}_{2}$ & 98.099 \\
\hline 6 & 8.655 & 1-Methoxy-1-buten-3-yne & 1.67 & $\mathrm{C}_{5} \mathrm{H}_{6} \mathrm{O}$ & 82.102 \\
\hline 7 & 9.779 & 2-Pentyn-1-ol & 4.87 & $\mathrm{C}_{5} \mathrm{H}_{8} \mathrm{O}$ & 84.118 \\
\hline 8 & 11.407 & 1,5-Heptadiene-3-yne & 2.23 & $\mathrm{C}_{7} \mathrm{H}_{8}$ & 92.141 \\
\hline 9 & 12.764 & 2-Propenenitrile & 1.04 & $\mathrm{C}_{3} \mathrm{H}_{3} \mathrm{~N}$ & 53.064 \\
\hline 10 & 13.191 & 4-Cyclopentene-1,3-diol, trans & 0.96 & $\mathrm{C}_{5} \mathrm{H}_{8} \mathrm{O}_{2}$ & 100.12 \\
\hline 11 & 13.888 & 2-Propenenitrile & 1.19 & $\mathrm{C}_{3} \mathrm{H}_{3} \mathrm{~N}$ & 53.064 \\
\hline 12 & 14.974 & Carbonic acid, but-3-yn-1-yl-undecyl ester. & 2.16 & $\mathrm{C}_{16} \mathrm{H}_{28} \mathrm{O}_{3}$ & 268.391 \\
\hline 13 & 16.719 & N'-(Diaminomethylidene)butane-hydrazide & 3.95 & $\mathrm{C}_{9} \mathrm{H}_{20} \mathrm{~N}_{40}$ & 200.28 \\
\hline 14 & 39.863 & 6-Methyl-triazolo (4,3-b))1,2,4)-triazine & 1.11 & $\mathrm{C}_{5} \mathrm{H}_{5} \mathrm{~N}_{5}$ & 135.13 \\
\hline 15 & 42.344 & 1,5-Hexadiyne & 1.43 & $\mathrm{C}_{6} \mathrm{H}_{6}$ & 78.114 \\
\hline 16 & 46.299 & 3-Dimethyl(Phenyl) Silyloxytridecan & 2.92 & - & - \\
\hline 17 & 50.214 & D-Allose & 19.93 & $\mathrm{C}_{6} \mathrm{H}_{12} \mathrm{O}_{6}$ & 180.156 \\
\hline 18 & 50.873 & $\begin{array}{c}\text { 6-(1-Adamatylamino)-2,4,5-trichloronicot- } \\
\text { inonitrile }\end{array}$ & 1.50 & $\mathrm{C}_{6} \mathrm{H}_{16} \mathrm{O}_{2}$ & 356.672 \\
\hline
\end{tabular}


Evaluation of Bioactive Compounds in Moringa oleifera Flower Using Gas Chromatography Mass Spectrometry/Fourier Transform Infrared Spectroscopy: The Need for Good Postharvest Handling

\begin{tabular}{|l|c|c|c|c|c|}
\hline 19 & 53.316 & Allyl fluoride & 1.06 & $\mathrm{C}_{3} \mathrm{H}_{5} \mathrm{~F}$ & 60.071 \\
\hline 20 & 55.215 & 1,12-Tridecadiene & 29.78 & $\mathrm{C}_{13} \mathrm{H}_{24}$ & 180.335 \\
\hline 21 & 56.068 & 3-Methyl-1-hexyne & 1.93 & $\mathrm{C}_{7} \mathrm{H}_{12}$ & 96.173 \\
\hline 22 & 56.727 & 1-propanol & 0.93 & $\mathrm{C}_{3} \mathrm{H}_{8} \mathrm{O}$ & 60.095 \\
\hline 23 & 62.349 & Guanidine-methyl & 0.82 & $\mathrm{C}_{2} \mathrm{H}_{7} \mathrm{~N}_{3}$ & 73.10 \\
\hline
\end{tabular}

Table 1: Gas Chromatography Mass Spectrometry analysis of bioactive compounds in methanol extract of fresh Moringa oleifera flowers stored at ambient temperature.

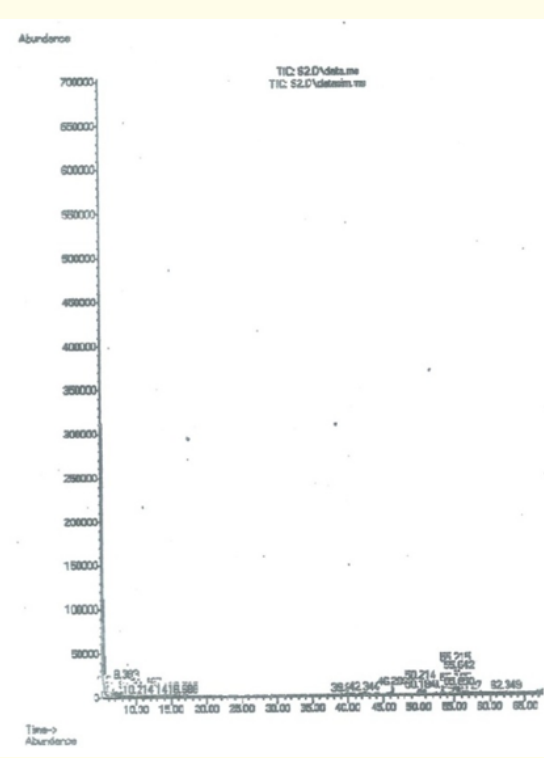

Figure 1: Gas Chromatography - Mass Spectrometry Chromatogram of Methanol extract of freshly harvested Moringa oleifera flowers stored at ambient temperature.

Table 2 shows results of bioactive compounds in dichloromethane/methanol extract of freshly harvested and processed Moringa oleifera flowers. The most abundant compounds found in this fraction include hexadecanoic acid methyl ester, 9,12-Octadecanoic acid methyl ester and 10,13-Octadecadienoic acid methyl ester. They had a relative abundance of $24.44,20.28$ and $20.28 \%$ respectively. These represent $65 \%$ of the compounds in hydrophobic fraction of bioactive compounds present in this extract. Hexadecanoic acid methyl ester has been reported to have antioxidant, hypocholesterolemic, anti-inflammatory activities [25] as well as antibacterial and antifungal effects [26]. 10,13-Octadecadieonic acid methyl ester has been reported to have many biological activities such as anti-inflammatory, hypocholesterolemic, cancer preventive, hepatoprotective, nematicide, insectifuge, anti-eczemic, anticancer, anti-arthritic, antihistaminic and anticoronary activities [27]. [28] reported 9,12-Octadecadienoic acid methyl ester to have anticancer activity.

Other compounds found in significant quantities include tetracosanoic acid methyl ester (7.24\%), methyl-18-methylnonadecanoate (4.92\%), cis-methyl-11-eicosenoate (4.36\%), trans-13-0ctadecenoic acid (2.93\%), docosanoic acid methyl ester (4.83\%), 1,3,5-Triazin-2(IH)-one-4,6-bis-(ethylamino) (2.19\%) and 2-Ethylbutyric acid, 2-hexyl ester (1.29\%).

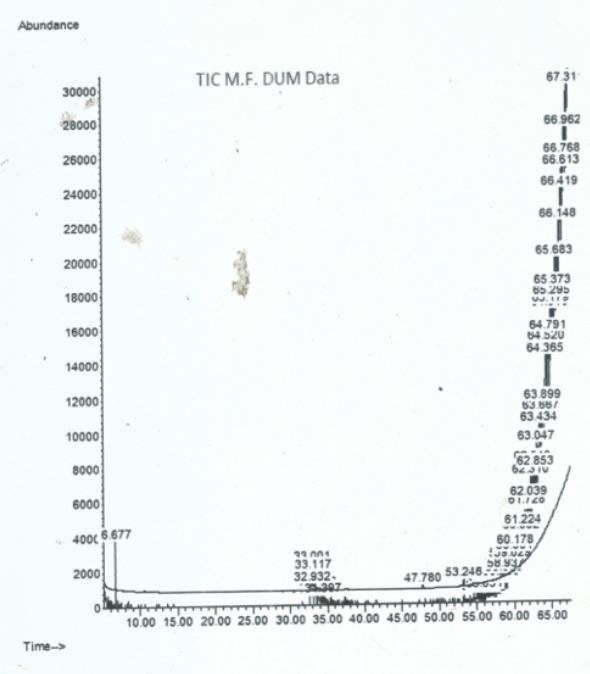

Figure 2: Gas Chromatography - Mass Spectrometry Chromatogram of Dichloromethane/Methanol extract of freshly harvested Moringa oleifera flower processed without storage at ambient temperature. 
Evaluation of Bioactive Compounds in Moringa oleifera Flower Using Gas Chromatography Mass Spectrometry/Fourier Transform Infrared Spectroscopy: The Need for Good Postharvest Handling

\begin{tabular}{|c|c|c|c|c|c|}
\hline $\begin{array}{l}\text { Peak } \\
\text { Number }\end{array}$ & $\begin{array}{l}\text { Retention } \\
\text { Time (Min) }\end{array}$ & Compound Name & $\begin{array}{c}\text { Relative } \\
\text { Abundance } \\
(\%)\end{array}$ & $\begin{array}{c}\text { Molecular } \\
\text { Formula }\end{array}$ & $\begin{array}{l}\text { Molecular } \\
\text { Weight }\end{array}$ \\
\hline 1 & 8.383 & 2-Pentene,3-Methyl, E- & 0.01 & $\mathrm{C}_{6} \mathrm{H}_{12}$ & 84.16 \\
\hline 2 & 15.594 & Octanoic acid, methyl ester & 0.15 & $\mathrm{C}_{9} \mathrm{H}_{18} \mathrm{O}_{2}$ & 158.23 \\
\hline 3 & 23.735 & 2,4-Decadienal & 0.11 & $\mathrm{C}_{10} \mathrm{H}_{16} \mathrm{O}$ & 152.23 \\
\hline 5 & 30.093 & Nonanoic acid, 9-oxo-methyl ester & 0.03 & $\mathrm{C}_{10} \mathrm{H}_{18} \mathrm{O}_{3}$ & 186.25 \\
\hline 6 & 33.738 & $\begin{array}{l}\text { Undecanoic acid, 10-methyl-, } \\
\text { methyl-1-ester }\end{array}$ & 0.09 & $\mathrm{C}_{13} \mathrm{H}_{26} \mathrm{O}_{2}$ & 214.34 \\
\hline 7 & 37.537 & Dodecanoic & 0.13 & $\mathrm{C}_{12} \mathrm{H}_{24} \mathrm{O}_{2}$ & 200.32 \\
\hline 8 & 41.801 & Methyl tetradecanoate & 0.05 & $\mathrm{C}_{15} \mathrm{H}_{30} \mathrm{O}_{2}$ & 242.40 \\
\hline 9 & 44.942 & Acetamide, N-(3-oxo-4-isi-xazolidinyl-, (R) & 0.02 & $\mathrm{C}_{5} \mathrm{H}_{8} \mathrm{~N}_{2} \mathrm{O}_{3}$ & 144.13 \\
\hline 10 & 48.392 & $\begin{array}{c}\text { Bicycle[4.1.0]heptane,-3-cyclopropyl,-7-carbomethoxy, } \\
\text { trans }\end{array}$ & 0.01 & $\mathrm{C}_{12} \mathrm{H}_{18} \mathrm{O}_{2}$ & 194.27 \\
\hline 11 & 49.594 & Hexadecanoic acid, methyl ester & 24.44 & $\mathrm{C}_{17} \mathrm{H}_{34} \mathrm{O}_{2}$ & 270.50 \\
\hline 12 & 51.067 & Bicyclopentylidene & 0.02 & $\mathrm{C}_{10} \mathrm{H}_{16}$ & 136.23 \\
\hline 13 & 52.424 & 3,4-Heptadiene & 0.04 & $\mathrm{C}_{7} \mathrm{H}_{12}$ & 96.17 \\
\hline 14 & 54.595 & 9,12-Octadecadienoic acid, methyl ester & 20.28 & $\mathrm{C}_{19} \mathrm{H}_{34} \mathrm{O}_{2}$ & 294.47 \\
\hline 15 & 56.882 & 10,13-Octadecadienoic acid, methyl ester & 20.28 & $\mathrm{C}_{19} \mathrm{H}_{34} \mathrm{O}_{2}$ & 294.47 \\
\hline 16 & 57.309 & trans-13-Octadecenoic acid & 2.93 & $\mathrm{C}_{18} \mathrm{H}_{34} \mathrm{O}_{2}$ & 282.50 \\
\hline 17 & 57.464 & Methyl-9-Cis.,11-trans.t,13-trans.- Octadecadienoate & 0.72 & $\mathrm{C}_{19} \mathrm{H}_{34} \mathrm{O}_{2}$ & 294.47 \\
\hline 18 & 57.658 & 10-Nonadecenoic acid, methyl ester & 0.04 & $\mathrm{C}_{20} \mathrm{H}_{38} \mathrm{O}_{2}$ & 310.50 \\
\hline 19 & 57.890 & 13-Octadecenal (Z)- & 0.02 & $\mathrm{C}_{18} \mathrm{H}_{34} \mathrm{O}$ & 266.468 \\
\hline 20 & 58.821 & 9,17-Octadecadienal, (Z) - & 0.77 & $\mathrm{C}_{18} \mathrm{H}_{32} \mathrm{O}$ & 264.40 \\
\hline 21 & 59.170 & Cis-Methyl 11-eicosenoate & 4.36 & $\mathrm{C}_{21} \mathrm{H}_{40} \mathrm{O}_{2}$ & 324.50 \\
\hline 22 & 59.674 & Methyl 18-methylnonadecanoate & 4.92 & $\mathrm{C}_{21} \mathrm{H}_{40} \mathrm{O}_{2}$ & 326.60 \\
\hline 23 & 59.945 & Dichloroacetic acid, undec-2-enyl ester & 0.16 & $\mathrm{C}_{13} \mathrm{H}_{22} \mathrm{Cl}_{2} \mathrm{O}_{2}$ & 281.22 \\
\hline 24 & 60.061 & 6-Nitroundec-5-ene & 0.25 & $\mathrm{C}_{11} \mathrm{H}_{21} \mathrm{NO}_{2}$ & 199.29 \\
\hline 25 & 60.294 & 2,4-Hexadiene-1,6-dimethoxy, (E,E) & 0.09 & $\mathrm{C}_{8} \mathrm{H}_{14} \mathrm{O}_{2}$ & 142.20 \\
\hline 26 & 60.410 & Z-2-Dodecenol & 0.05 & $\mathrm{C}_{12} \mathrm{H}_{24} \mathrm{O}$ & 184.32 \\
\hline 27 & 60.565 & Oxirane undecanoic acid, 3-pentyl 1-methyl ester, cis- & 0.05 & $\mathrm{C}_{19} \mathrm{H}_{36} \mathrm{O}_{3}$ & 312.49 \\
\hline 28 & 60.682 & $\begin{array}{l}\alpha \text {-d-,Glucose-4,6-0-isopropylidiene-1-0-methyl-6-0 } \\
\text { [4-bromobenzenesulfonate] }\end{array}$ & 0.09 & $\mathrm{C}_{16} \mathrm{H}_{21} \mathrm{BrO}_{8}$ & 453.30 \\
\hline 29 & 61.031 & Heneicosanoic acid, methyl ester & 0.25 & $\mathrm{C}_{22} \mathrm{H}_{44} \mathrm{O}_{2}$ & 340.60 \\
\hline 30 & 61.535 & Methyl-2-octylcyclopropene-1-octanoate & 0.76 & $\mathrm{C}_{20} \mathrm{H}_{36}$ & 308.49 \\
\hline 31 & 61.806 & 2H-Azepin-2-one,hexahydro-7-methyl & 0.13 & $\mathrm{C}_{7} \mathrm{H}_{13} \mathrm{NO}$ & 127.18 \\
\hline 32 & 62.271 & Cyclododecanol,1-aminomethyl & 0.20 & $\mathrm{C}_{13} \mathrm{H}_{27} \mathrm{NO}$ & 213.36 \\
\hline 33 & 62.659 & Docosanoic acid, methyl ester & 4.83 & $\mathrm{C}_{23} \mathrm{H}_{46} \mathrm{O}_{2}$ & 354.61 \\
\hline
\end{tabular}


Evaluation of Bioactive Compounds in Moringa oleifera Flower Using Gas Chromatography Mass Spectrometry/Fourier Transform Infrared Spectroscopy: The Need for Good Postharvest Handling

\begin{tabular}{|l|c|c|c|c|c|}
\hline 34 & 63.046 & 8-(2-Octanoylcyclopropyl)Octanoic acid, methyl ester & 0.61 & \\
\hline 35 & 63.318 & 1,3,5-Triazin-2(1H)-one-4,6-bis-(ethylamino) & 2.19 & $\mathrm{C}_{7} \mathrm{H}_{13} \mathrm{~N}_{5} \mathrm{O}$ & 183.21 \\
\hline 36 & 63.938 & Tricosanoic acid, methyl ester & 0.65 & $\mathrm{C}_{24} \mathrm{H}_{48} \mathrm{O}_{2}$ & 368.6 \\
\hline 37 & 64.093 & Pentanoic acid, 2-methyl-,1-methyl propyl ester & 0.25 & $\mathrm{C}_{10} \mathrm{H}_{20} \mathrm{O}_{2}$ & 172.26 \\
\hline 38 & 64.869 & 2-Ethylbutyric acid, 2-hexyl ester & 1.29 & $\mathrm{C}_{12} \mathrm{H}_{24} \mathrm{O}_{2}$ & 200.32 \\
\hline 39 & 65.411 & Tetracosanoic acid, methyl ester & 7.24 & $\mathrm{C}_{25} \mathrm{H}_{50} \mathrm{O}_{2}$ & 382.70 \\
\hline 40 & 65.915 & Fumaric acid,isohexyl-4-methyl pent-2-yl ester & 0.61 & $\mathrm{C}_{16} \mathrm{H}_{28} \mathrm{O}_{4}$ & 284.31 \\
\hline 41 & 66.032 & Octanoic acid, 2-pentadecyl ester & 0.11 & $\mathrm{C}_{23} \mathrm{H}_{46} \mathrm{O}_{2}$ & 354.6 \\
\hline 42 & 66.574 & Pentacosanoic acid, methyl ester & 0.16 & $\mathrm{C}_{26} \mathrm{H}_{52} \mathrm{O}_{2}$ & 316.7 \\
\hline 43 & 67.233 & Cyclohexanol,1R-4-acetamido-2,3-cis epoxy & 0.03 & - & - \\
\hline
\end{tabular}

Table 2: Gas Chromatography Mass Spectrometry analysis of bioactive compounds in dichloromethane/methanol extract of freshly harvested and processed Moringa oleifera without storage at ambient temperature.

Table 3 shows results of dichloromethane/methanol extract of fresh Moringa oleifera flowers sample stored at ambient temperature. The most abundant compounds were hydrazine-1,1-dimethyl, acetic acid, urea and isobutylamine and they had relative abundance of $19.87 \%, 7.45 \%, 10.42 \%$ and $10.69 \%$ respectively. These account for $58.5 \%$ of bioactive compounds in this hydrophobic extract. Results showed the presence of degradation products of amino acids and nitrogenous compounds. [9] reported M. oleifera flower to have higher amino acid content than the leaves or seeds. Some of the compounds from this extract such as hydrazine-1,1dimethyl and its analogue hydrazine-1,2-dimethyl have deleterious biological activities and were found in appreciable quantities. They are derived from hydrazine. These two compounds are classified as alkylating agents and are able to introduce alkyl radicals into biologically active molecules thereby prevent their proper functioning and so can be carcinogenic, mutagenic, teratogenic and possess immunosuppressant actions [29,30]. Acetic acid has been reported to have acidulant, antibacterial, mucolytic, osteolytic, perfumery, pesticide, protisticide, spermicide, ulcerogenic and verrucolytic activities [31]. Isobutylamine is a monoalkylamine containing a primary aliphatic amine group. There are applications for isobutylamine as it can be used as food additive such as flavoring agent or flavor adjuvant [32]. Urea is used as food additive in some sugar free chewing gum to adjust the texture and its addition up to $3 \%$ cannot be of toxicological concern [33]. However, postharvest handling in a cellophane bag at ambient temperature resulted to a 'build up' of urea to a high concentration $(10.42 \%)$. This could have occurred due to activities of endogenous enzymes on amino acids present in the Moringa oleifera flowers. The amount of urea formed could be of toxicological concern if ingested. Another compound found in significant quantity was thiirane and it has been reported to have anticancer activity [34].

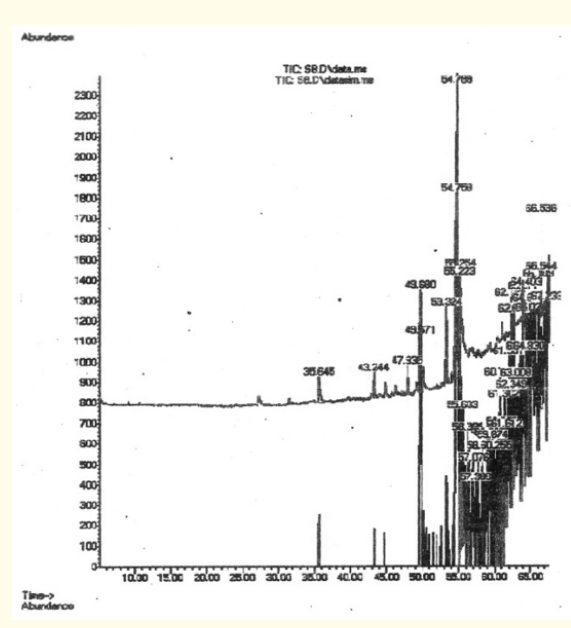

Figure 3: Gas Chromatography - Mass Spectrometry Chromatogram of Dichloromethane / Methanol Extract of Moringa oleifera flower stored at ambient temperature. 
Evaluation of Bioactive Compounds in Moringa oleifera Flower Using Gas Chromatography Mass Spectrometry/Fourier Transform Infrared Spectroscopy: The Need for Good Postharvest Handling

\begin{tabular}{|l|c|c|c|c|c|}
\hline $\begin{array}{l}\text { Peak } \\
\text { Number }\end{array}$ & $\begin{array}{c}\text { Retention } \\
\text { Time (Min) }\end{array}$ & Compound Name & $\begin{array}{c}\text { Relative } \\
\text { Abundance } \\
\text { (\%) }\end{array}$ & $\begin{array}{c}\text { Molecular } \\
\text { Formula }\end{array}$ & $\begin{array}{c}\text { Molecular } \\
\text { Weight }\end{array}$ \\
\hline 1 & 49.671 & Acetic acid & 7.45 & $\mathrm{C}_{2} \mathrm{H}_{4} \mathrm{O}_{2}$ & 60.052 \\
\hline 2 & 54.789 & Hydrazine-1,1-dimethyl & 19.87 & $\mathrm{C}_{2} \mathrm{H}_{8} \mathrm{~N}_{2}$ & 60.10 \\
\hline 3 & 55.254 & Thiirane & 8.76 & $\mathrm{C}_{2} \mathrm{H}_{4} \mathrm{~S}$ & 60.114 \\
\hline 4 & 55.603 & Urea & 10.42 & $\mathrm{CH}_{4} \mathrm{~N}_{2} \mathrm{O}$ & 60.056 \\
\hline 5 & 57.386 & N-Ethylformamide & 2.25 & $\mathrm{C}_{3} \mathrm{H}_{7} \mathrm{NO}$ & 73.095 \\
\hline 6 & 58.976 & Acetic acid & 10.07 & $\mathrm{C}_{2} \mathrm{H}_{4} \mathrm{O}_{2}$ & 60.052 \\
\hline 7 & 60.992 & Carbonyl sulfide & 2.39 & $\mathrm{COS}_{2}$ & 60.07 \\
\hline 8 & 61.612 & Hydrazine-1,2-dimethyl & 7.79 & $\mathrm{C}_{2} \mathrm{H}_{8} \mathrm{~N}_{2}$ & 60.10 \\
\hline 9 & 61.961 & Propanamide & 9.20 & $\mathrm{C}_{3} \mathrm{H}_{7} \mathrm{NO}$ & 73.095 \\
10 & 62.349 & Isobutyl amine & 10.69 & $\mathrm{C}_{4} \mathrm{H}_{11} \mathrm{~N}$ & 73.139 \\
11 & 63.977 & 2-Butanamine & 4.95 & $\mathrm{C}_{4} \mathrm{H}_{11} \mathrm{~N}$ & 73.139 \\
12 & 64.636 & O-n-Propylhydroylamine & 3.44 & $\mathrm{C}_{3} \mathrm{H}_{9} \mathrm{NO}$ & 75.11 \\
13 & 67.233 & Silamamine,N-Silyl & 2.72 & $\mathrm{HNSi}_{2}$ & 71.185 \\
\hline
\end{tabular}

Table 3: Gas Chromatography Mass Spectrometry analysis of bioactive compounds in dichloromethane/methanol extract of freshly harvested Moringa oleifera flower and kept in cellophane bag at ambient temperature.

Functional groups enumerated by Fourier Transform Infra Red (FTIR) studies correlate to compounds elucidated by GCMS. It is used as a supportive tool in qualitative bioactive compound screening. Table 4-6 shows FTIR spectroscopy results of the various extracts. The polarity of the respective solvents played a major role in extracting more specific bioactive compounds with methanol extracting more of the carboxylic acid compounds, alcohols, aldehydes, alkanes, alkenes, alkynes and nitrogen containing compounds, Dichloromethane/methanol extracted more of the fatty acids, fatty acid methyl esters, amine, alkane, alkene and cyclic compounds.

\begin{tabular}{|l|c|c|c|c|c|}
\hline S/No & $\begin{array}{c}\text { Band } \\
\text { Position } \\
\text { (cm-) }^{-}\end{array}$ & $\begin{array}{c}\text { Wave } \\
\text { Intensity }\end{array}$ & Bond & $\begin{array}{c}\text { Functional } \\
\text { group } \\
\text { assignment }\end{array}$ & $\begin{array}{c}\text { Group } \\
\text { frequency }\end{array}$ \\
\hline 1 & 3268.9 & 51.453 & O-H broad & $\begin{array}{c}\text { Hydroxy } \\
\text { group, } \\
\text { H-bonded }\end{array}$ & $3600-3200$ \\
\hline & 2929.7 & 81.688 & O-H & $\begin{array}{c}\text { Aliphatic } \\
\text { primary amine }\end{array}$ & $3330-3250$ \\
\hline 2 & stretching & $\begin{array}{c}\text { Intermolecular } \\
\text { bonded } \\
\text { alcohol }\end{array}$ & $3200-2700$ \\
\hline
\end{tabular}

\begin{tabular}{|c|c|c|c|c|c|}
\hline 3 & 2855.1 & 87.952 & $\mathrm{C}-\mathrm{H}$ & Aldehyde & $2860-2800$ \\
\hline 4 & 1640.0 & 70.354 & $\mathrm{C}-\mathrm{N}$ & $\begin{array}{l}\text { Organic } \\
\text { nitrates }\end{array}$ & $1640-1620$ \\
\hline 5 & 1453.7 & 84.045 & $C=E C$ & $\begin{array}{c}\text { Aromatic ring } \\
\text { stetch }\end{array}$ & $1510-1450$ \\
\hline 6 & 1408.9 & 82.798 & $C=0$ & $\begin{array}{c}\text { Carboxylate/ } \\
\text { caboxylic acid } \\
\text { salt }\end{array}$ & $1420-1300$ \\
\hline \multirow[t]{3}{*}{7} & 1013.8 & 57.085 & $>\mathrm{CH}$ & Methyne & $1300-700$ \\
\hline & & & $>\mathrm{CH}_{2^{\mathrm{a}}}$ & $\begin{array}{c}\text { Cyclohexane } \\
\text { ring vibrations }\end{array}$ & $1055-1000$ \\
\hline & & & GF stretch & $\begin{array}{c}\text { Aliphatic } \\
\text { fluoro } \\
\text { compound }\end{array}$ & $1150-1000$ \\
\hline
\end{tabular}

Table 4: Fourier Transform Infrared Spectroscopy results on methanol extract of freshly harvested Moringa oleifera flowers stored at ambient temperature. 


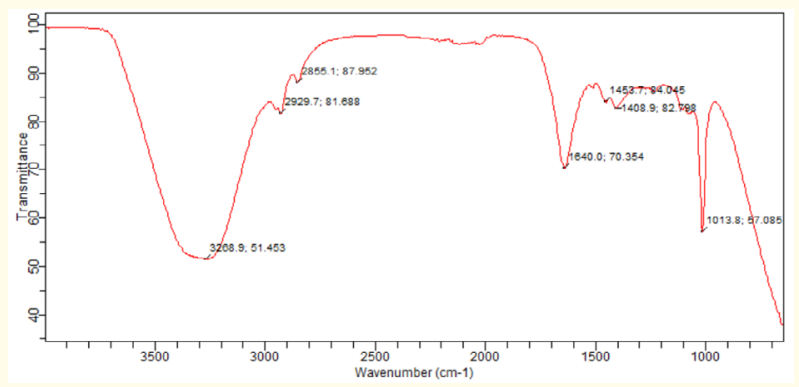

Figure 4: FTIR Spectrograph of methanol extract of freshly harvested Moringa oleifera flowers stored at ambient temperature.

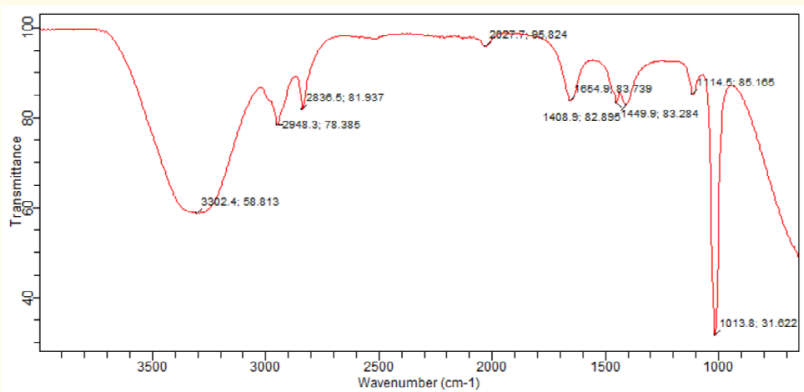

Figure 5: FTIR Spectrograph of dichloromethane/methanol extract of freshly harvested Moringa oleifera flowers.

\begin{tabular}{|c|c|c|c|c|c|}
\hline S/No & $\begin{array}{c}\text { Band Position } \\
\left(\mathrm{cm}^{-}\right)\end{array}$ & Wave Intensity & Bond & Functional group assignment & Group frequency \\
\hline 1 & 3302.4 & 58.813 & O-H broad & Hydrogen bonded alchohols & $3600-3200$ \\
\hline & & & $\mathrm{N}-\mathrm{H}$ & Phenol, OH-group & \\
\hline 2 & 2948.3 & 78.385 & $\begin{array}{l}\text { O-H stretch } \\
\text { N-H } \\
\text { C-H stretch }\end{array}$ & $\begin{array}{l}\text { Intermolecular bonded hydroxl group, } \\
\text { Amine } \\
\text { Alkane }\end{array}$ & $\begin{array}{l}3200-2700 \\
3000-2800 \\
3000-2850\end{array}$ \\
\hline 3 & 2836.5 & 81.937 & $\mathrm{C}-\mathrm{H}, \mathrm{O}-\mathrm{CH}_{3}$ & Methoxy, methyl ether & $2850-2815$ \\
\hline 4 & 2027.7 & 95.824 & Unknown & Unknown & - \\
\hline 5 & 1654.9 & 83.739 & $\begin{array}{l}\mathrm{C}=\mathrm{O} \\
\mathrm{C}=\mathrm{C}\end{array}$ & $\begin{array}{c}\text { Saturated amide } \\
\text { Alkene }\end{array}$ & $\begin{array}{l}1680-1620 \\
1680-1620\end{array}$ \\
\hline 6 & 1449.9 & 83.284 & $\mathrm{C}=\mathrm{GC}$ Weak & Aromatic & $1510-1450$ \\
\hline 7 & $\begin{array}{l}1408.9 \\
1114.5\end{array}$ & 82.895 & $\begin{array}{l}\text { O-H bend } \\
\begin{array}{l}\mathrm{C}-\mathrm{O} \\
>\mathrm{CH}_{2}\end{array}\end{array}$ & $\begin{array}{c}\text { Carboxylic acid } \\
\text { Phenol, tertiary alchohol } \\
\text { Secondary alchohol } \\
\text { Cyclohexane ring vibrations }\end{array}$ & $\begin{array}{l}1440-1395 \\
1410-1310 \\
1124-1087 \\
1055-1000\end{array}$ \\
\hline 9 & 1013.8 & 31.622 & C-O Stretch & Esters, Alkoxy & $1150-1000$ \\
\hline
\end{tabular}

Table 5: Fourier Transform Infrared Spectroscopy results of Dichloromethane/methanol extract of freshly harvested Moringa oleifera flowers.

\begin{tabular}{|c|c|c|c|c|c|}
\hline S/No & $\begin{array}{c}\text { Band Position } \\
\left(\mathrm{cm}^{-}\right)\end{array}$ & Wave Intensity & Bond & Functional group assignment & Group frequency \\
\hline \multirow[t]{2}{*}{1} & 3268.9 & 51.453 & O-H broad & Hydroxy group,H-bonded & $3600-3200$ \\
\hline & & & $\mathrm{N}-\mathrm{H}$ & Aliphatic primary amine & $3330-3250$ \\
\hline 2 & $\begin{array}{l}2948.3 \\
2836.5\end{array}$ & $\begin{array}{l}77.792 \\
81.385\end{array}$ & $\begin{array}{l}\text { O-H stretch } \\
\quad \mathrm{N}-\mathrm{H} \\
\text { C-H stretch } \\
\text { C-H stretch, }\end{array}$ & $\begin{array}{l}\text { Intermolecular bonded hydroxl group, } \\
\text { Amine } \\
\text { Alkane } \\
\text { Methoxy }\end{array}$ & $\begin{array}{l}3200-2700 \\
3000-2800 \\
3000-2850 \\
2850-2815\end{array}$ \\
\hline 3 & & & $\mathrm{O}-\mathrm{CH}_{3}$ & Methyl ether & \\
\hline
\end{tabular}


Evaluation of Bioactive Compounds in Moringa oleifera Flower Using Gas Chromatography Mass Spectrometry/Fourier Transform Infrared Spectroscopy: The Need for Good Postharvest Handling

\begin{tabular}{|l|l|l|c|c|c|}
\hline 4 & 1654.9 & 83.848 & $\mathrm{~N}-\mathrm{H}$ & Amide & $1680-1630$ \\
5 & 1449.9 & 82.900 & $\mathrm{C}=\mathrm{C}-\mathrm{C}$ & Aromatic ring stretch & $1510-1450$ \\
6 & 1408.9 & 82.384 & $\mathrm{C}=\mathrm{O}$ & Carboxylate/Carboxylic acid salt & $1420-1300$ \\
7 & 1110.7 & 84.466 & $\mathrm{~S}-\mathrm{O}-\mathrm{C}$ & Organic siloxane/ Silicone & $1110-1080$ \\
8 & 1013.8 & 30.196 & $>\mathrm{CH}_{2}$ & Cyclohexane ring vibrations & $1055-1000$ \\
& & & $\mathrm{C}-\mathrm{O}$ & Esters, Alkoxy & $1150-1000$ \\
\hline
\end{tabular}

Table 6: Fourier Transform Infrared Spectroscopy results of Dichloromethane/methanol extract of fresh flowers of Moringa oleifera kept at ambient temperature in a cellophane bag.

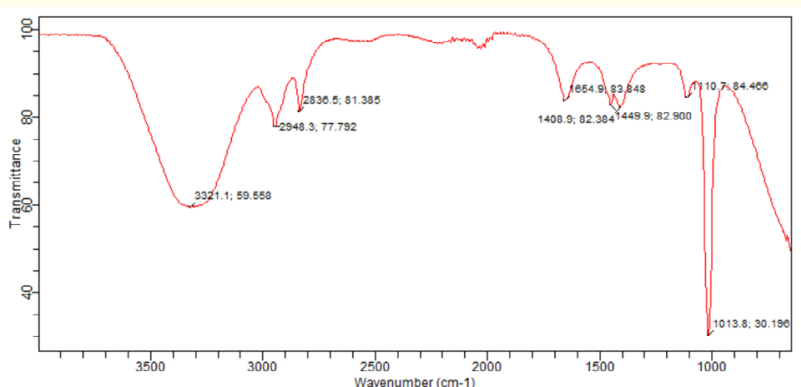

Figure 6: FTIR Spectrograph of dichloromethane/methanol extract of freshly harvested Moringa oleifera flowers stored at ambient temperature.

\section{Bibliography}

1. Navarro-Gonzalez I., et al. "Nutritional Composition and antioxidant capacity in edible flowers: Characteristics of phenolic compounds by HPLC-DAD-ESI/MS". International Journal of Molecular Science 16.1 (2015): 805-822.

2. Alasalvar C., et al. "Compositional, nutritional and functional characteristics of instant teas produced from low and highquality black teas". Journal of Agriculture and Food Chemistry 61 (2013): 7529-7536.

3. Padayachee B and Baijnath H. "An overview of the medicinal importance of Moringaceae". Journal of Medicinal Plants Research 6 (2012) :5831-5839.

4. Khalafalla MM., et al. "Active principle from Moringa oleifera Lam. Leaves effective against two leukemia and heptocacinoma”. African Journal of Biotechnology 9.49 (2010): 8467-8471.

5. Leone A., et al. "Moringa oleifera seeds and oil: Characteristics and uses for human health". International Journal of Molecular Sciences (2016): 2141.

\section{Conclusion}

The effect of postharvest handling of Moringa oleifera flower in cellophane paper bag and kept at ambient temperature resulted to the formation of deleterious compounds such as hydrazine-1,1dimethyl and its analogue hydrazine-1,2-dimethyl which are carcinogenic. There was also a postharvest build-up of urea and acetic acid. Some compounds from the methanol extract as well as dichloromethane/methanol extract of the freshly harvested flowers have been reported to have beneficial effects for wellness and good health. This research work provides information on the need for efficient postharvest handling such as cold storage immediately after harvest prior to processing so as to avoid the build-up or formation of deleterious bioactive compounds.

6. Puri V. "Floral Anatomy of the Moringaceae with special reference to gynoecium constitution". Proceedings of the national Institute of Sciences of India Studies in floral anatomy II, national Institute of Sciences of India (1942).

7. Decraene LR., et al. "Floral Development and anatomy of Moringa oleifera (Moringaceae). What is the evidence for a Capparalean or Sapindalean Affinity?" Annals of Botany 82.3 (1998): 273-284.

8. Parrota JA. "Moringa oleifera Lam. 1785". In: A Roloff, H Weisgerber, U Lang, B Stimm (Editors). Enzyklopadie der Holozgewachse, Hanbuch und Allas der Dendrologi. Wiley VohVerlag GmbH and Co KGA, Weinheim (2009): 1-9.

9. Amaechi NC., et al. Comparative evaluation of amino acids in leaves, seeds and flowers of Moringa oleifera: Potentials in combating malnutrition. "Proceedings of the $2^{\text {nd }}$ International Conference on Food Security and Hidden Hunger, Faculty of Agriculture, Alex Ekwueme Federal University, Ndufu-Alike, Ebonyi State, Nigeria" (2018): 220-225. 
10. Skrajda MN. "Phenolic compounds and antioxidant activity of edible flowers". Journal of Education, Health and Sport 7.8 (2007): 946-956.

11. Kalappurayil TM and Joseph BP. "A review of Pharmacognostical studies on Moringa oleifera Lam. Flowers". Pharmacognosy Journal 9.1 (2017): 1-7.

12. Kopka J. "Gas chromatography-mass spectrometry". Agricultural biotechnology 57 (2006): 3-20.

13. Mariswamy, Y., et al. "FTIR Spectroscopic studies on Aerva lanata (1.) Juss. ex schult". Asian Journal of Pharmaceutical and Clinical Research 5.2 (2012): 82-86.

14. Hemalatha R., et al. "Phytochemical composition, GC-MS analysis, in vitro antioxidant and antibacterial potential of clove flower bud (Eugenia caryophyllus) methanol extract". Journal of Food Science and Technology 53.2 (2016): 1189-1198.

15. Orji OU., et al. "Investigations of phytochemical and nutritional composition of Ruspolia hypocrateriformis leaf". IDOSR Journal of Applied Sciences 2.2 (2017): 7-81.

16. Yugndhar $\mathrm{P}$ and Savithramma N. "Spectroscopic and chromatographic exploration of different phytochemical and mineral contents from Syzygium alternifolim (Wt.) Walp. an endemic, endangered medicinal tree taxon". Journal of Applied Pharmaceutical Science 7.1 (2017): 73-85.

17. Sasidharan S., et al. "Extraction, isolation and characteristics of bioactive compounds from plants extracts". African Traditional Complementary and Alternative Medicine 8.1 (2011): 1-10.

18. Inbathamizh L and Padmini E. "Gas chromatography Mass Spectrometry analysis of methanol extract of Moringa oleifera flower". International Journal of Chemical and Analytical Sciences 3.5 (2012): 1394-1397.

19. Ikpeazu OV., et al. "Preliminary studies on the secondary metabolites of Bucbhoziaz oriacea (Wonderful kola) seed ethanol extract by GCMS analysis". International Journal of Research in Engineering and Applied Sciences 7.3 (2017): 17-26.

20. Ishihara Y., et al. "Antioxidant properties of rare sugar D-Allose: Effects of mitochondrial reactive oxygen species production in Neuro2a cells". Journal of Biosciences and Bioengineering 116.6 (2011): 638-642.

21. Miyawaki Y., et al. "D-allose ameliorates cisplatin-induced nephrotoxicity in mice". Tohoku Journal of Experimental Medicine 228.3 (2012): 215-221.
22. Jeong RU., et al. "Effect of D-allose on prostate cancer cell lines: Phospholipid profiling by nanoflow liquid chromatography tandem mass spectrometry". Analytical and Bioanalytical Chemistry 401.2 (2011): 689-698.

23. Stevenson DG., et al. "Oil and tocopherol content and composition of Pumpkinseed oil in 12 cultivars". Journal of Agricultural and Food Chemistry 55 (2007): 4005-4013.

24. Igwe OU and Udofia DE. "Secondary metabolites of the culticular abdominal glands of variegated grasshopper (Zonocerus variegatus L.)". International Journal of Spectroscopy (2015): 6.

25. Belakhdah G., et al. "Determination of some bioactive chemical constituents from Thesium humile Vahl". Journal of Material and Environmental Sciences 6.10 (2015): 2778-2783.

26. Chandrasekaran M., et al. "Antibacterial and antifungal efficacy of fatty acid methyl esters from leaves of Sesuvium portulacastrum L". European Review for Medical and Pharmacological Sciences 15.7 (2011): 775-780.

27. Krishnamoorthy K and Subramanaim P. "Phytochemical profiling of leaf stem and tuber parts of Solena amplexicaulis (Lam) Gandhi using GC-MS". International Scholarly Research Notices (2014) 567409.

28. Yu FR., et al. "Isolation and characterization of methyl esters and derivatives from Euphorbia kanusi (Euphorbiaceae) and their inhibitory effects on the human SGC-7901 cells". Journal of Pharmacognosy and Pharmacetical Sciences 8 (2005): 528535.

29. Preston RJ and Ross JA. "DNA-Reactive agents". Comprehensive Toxicology (2ndEdition) 1 (2010): 349-360.

30. Forester SC and Lambert JD. "The Role of Antioxidant versus pro-oxidant effects of green tea polyphenols in cancer prevention". Molecular Nutrition and Food Research 55.6 (2011): 844854.

31. Yugandhar P and Savithramma N. "Spectroscopic and chromatrographic exploration of different phytochemical and mineral contents from Syzygium alternifolim (Wt) Walp. an endemic endangered medicinal tree taxon". Journal of Applied Pharmaceutical Science 7.1 (2017): 73-85.

32. "Evaluations of the Joint FAO/WHO Expert Committee on Food Additives (JECFA)". Food safety and quality- Isobutylamine: Specifications for flavorings (2005). 
33. Olsen P. "Urea”. Summary of Evaluations Performed by the Joint FAO/WHO Expert Committee on Food Additives/International Programme on Chemical Safety. TRS 837 JECFA 41/28. (2005).

34. Asif M. "Biological overview of thiirane derivatives". SOP Transactions on Applied Chemistry 1.1 (2014): 1-10.

Volume 5 Issue 12 December 2021

(c) All rights are reserved by Amaechi NC. 